\title{
The International Centre for Evidence-Based Periodontal Health
}

\author{
Ian Needleman", David Moles ${ }^{2}$ \\ ${ }^{1}$ Director, International Centre for Evidence-Based Periodontal Health, Eastman Dental Institute, London, UK \\ ${ }^{2}$ Deputy Director, International Centre for Evidence-Based Periodontal Health, Eastman Dental Institute, \\ London, UK
}

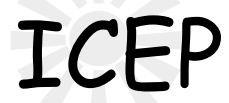

\begin{abstract}
We were delighted to launch the International Centre for Evidence-Based Periodontal Health (ICEP) at the Evidence-Based Dentistry conference in Boston, USA in January 2003. This is the culmination of a great deal of work over the last few years. The centre's mission is to enable research transfer for periodontal health. Here we briefly explain why the centre is needed, what it will do and how we hope that it will help to improve periodontal health.

Evidence-Based Dentistry (2003) 4, 2. doi:10.1038/sj.ebd.6400165
\end{abstract}

Good oral health requires a partnership between the well-developed skills and judgement of the clinician and the preferences and values of the patient. Evidence-based dentistry illuminates this partnership with the best available evidence. The greatest need for evidencebased dentistry is where there is uncertainty about how best to improve or maintain health and well-being. This uncertainty might arise from an apparent lack of answers to healthcare questions or, commonly, it is experienced where there are conflicting recommendations. Periodontology has its fair share of both and there is a strong international climate and desire to tackle these problems.

Having recognised that there is uncertainty, it is crucial that we develop some form of systematic evidence-base as an objective tool with which to examine the gaps in our understanding. By putting together all that is known about a topic and at the same time stripping away some of the misconceptions and biases that tend to collect around it, we should be more confident that we are armed with the best information to aid our decisions.

As practitioners we have seen many new treatment possibilities introduced.
Interventions like local antibiotics as an adjunct to nonsurgical therapy have produced conflicting results consequently when they might be used remains confusing to patients and to us. There are also much more fundamental questions to investigate, such as those concerning which methods are most effective in preventing the development of destructive periodontitis.

So, what will ICEP do? We aim to produce the most objective evidence for the prevention, diagnosis and treatment of periodontal diseases. This systematic evidence-base can then be used to inform the clinical decisions of those of us involved in health care, whether providing this to the individual in a practice or clinic, or to a community where population strategies are appropriate.

These overviews cannot ever be used as cookbook directives on how to practise. As an illustration of why not, consider the management of deep periodontal pockets that are not responsive to nonsurgical therapy, especially where deterioration is occurring. Pocket-elimination surgery may well be the most effective therapy with regard to reducing pocket depth, at least in the short-term. But does this mean that all patients with such pockets should receive this therapy and would we be negligent if we did not provide it? Of course not, for two linked reasons. First, many patients will simply not want this type of treatment, especially near the front of their mouths. They could choose other options which, although less effective in probing-depth reduction, are preferable and may minimise recession. Secondly, although such treatments might be more effective at achieving one objective, they might be less effective for other outcomes such as attachment level or they might have adverse effects, as we have seen in the example we used above (ie, root exposure).

Although ICEP is based in the UK, its work will be international. Collaborations have already included work in North America, Europe and Asia and we hope to develop this further. As well as different geographical locations, we are keen to collaborate with different healthcare settings and we would be delighted to discuss possible research projects.

Finally, we will also continue to provide generic training in conducting systematic reviews in oral healthcare. We see this as crucial to expanding the development of a rigorous evidence-base in dentistry. To this end, we are running intensive 4-day courses in March and September 2003.

For more information please e-mail Ian Needleman (i.needleman@eastman. ucl.ac.uk). 